of the inhibition of the newly discovered pathway is that our mouse model has highly improved tolerance to glucose while requiring only a fraction of insulin for this regulation," says Ingenium's chief executive Michael Nehls. "In contrast to other insulinsensitizing effects, the mice do not become obese - on the contrary, they slim under a high-fat diet. We believe this could be a breakthrough in obesity/diabetes research," says Klaus Dembowsky, vice-president of drug discovery

\section{From genotype to phenotype}

Despite the increasing emphasis on proteomics in target identification, DNA microarray technology is still a powerful technique for identifying genes involved in susceptibility to diseases. Target discovery and identification should soon be benefiting from the DNA microarrays of the complete human genome now sold by several companies. First to market in July 2003 was NimbleGen in Madison, Wisconsin, with a chip containing 200,000 probes, with an average of five probes per gene. This was followed in October 2003 by the Human Genome U133 Plus 2.0 array from Affymetrix in Santa Clara, California, with 1.3 million probes and the ability to analyse the expression of about 47,000 different transcripts. The most recent human wholegenome chip, launched last month, comes from Agilent Technologies, in Palo Alto, California. Agilent's double-density-format chip represents about 41,000 genes and, with Agilent's ImaGene image-analysis software, is compatible with most commercial microarray scanners for $25 \times 75$-mm chips.

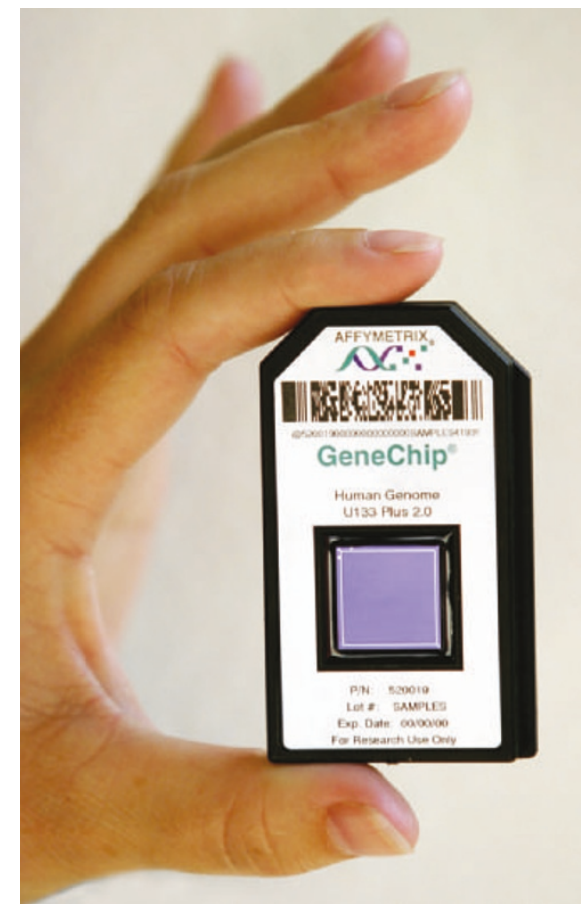

What it means to be human?

This open-platform approach, the company claims, will make it easier for scientists to move from their own homemade chips to the Agilent chip.

Microarrays are already widely used to study gene expression and to detect sequence features such as single-nucleotide polymorphisms (see Nature 422, 917-923; 2003). Future uses will include assays to study the many forms that a protein target may take as a result of alternative splicing. "For RNA analysis, over the next year, we will start to see assays for efficient splice variant analysis that will enable us to move from gene-specific analysis to transcript-specific analysis," says David Craford, vice-president of business development at Affymetrix. "More data are emerging to suggest that for many diseases this will be incredibly useful."

According to Greg Yap, senior marketing director in DNA analysis at Affymetrix, one of the newest and most exciting applications of microarrays to target identification is the possibility of genome-wide association studies of complex genetic diseases. "The experiments that geneticists really want to do are to find genes that cause complex diseases in unrelated clinical populations," says Yap. "Finding genes that cause a disease in a family makes a nice story, but finding genes causing a disease in the world makes a drug target." Studying genetically unrelated people is typically difficult, owing to the numbers of genetic markers per person that need to be studied. The advent of microarrays has made individual genotyping feasible, "making it realistic to compare the genomes of people with and without a disease at high-enough resolution to find potential drug targets", he says.

Pharmaceutical company Roche in Basel, Switzerland, is using the Affymetrix wholegenome expression arrays to identify genes that play key roles in disease pathways. "One approach for new target discovery that we use is to identify the genetic basis for disease susceptibility using a coupled genetic and genomic analysis," says Gary Peltz, head of genetics and genomics at Roche. "Having the complete genomes of several organisms (human, mouse and rat) available on chips is a major advance. It allows complex biology,

\title{
DRUG DISCOVERY IN REVERSE
}

Reversing the current drug-development path of 'target to drug to phenotype', chemogenomics, or chemical genomics, starts with a known drug that causes an interesting disease-relevant phenotype in vitro or in vivo, and then identifies the cellular target(s) for that drug. Chemogenomics is a powerful tool in target identification because you start with an active small molecule. By definition, any targets found are 'druggable' and are likely to be significant in the phenotype caused by the drug.

Seth Cohen, who recently moved from Millennium Pharmaceuticals to Caliper Life Sciences in Mountain View, California, likens chemogenomics to "reverse drug discovery". In other words, you take a drug compound with known effect and analyse in detail the result of exposure of the cell or organism to the compound. "This allows for a more precise understanding of the mechanism of action as well as potential causes of side effects. It also enables more intelligent subsequent screening of compounds with better, more relevant assay readouts," he says.

Iconix Pharmaceuticals in Mountain View, in collaboration with MDS Pharma Services of Lincoln, Nebraska, applies chemogenomics to the study of comprehensive responses of rats to treatment by a compound. Iconix uses CodeLink microarrays from Amersham Bioscience in Piscataway, New Jersey, which carry 10,000 probes for rat genes and expressed sequence tags, to measure gene expression in tissues from rats that have been treated with different drugs. MDS and Iconix have identified more than 250 clusters of genes whose expression changes in response to 600 drugs. "These clusters of genes, or 'drug signatures', describe and predict many specific mechanisms of action of the drug in the whole animal without the caveats of in vitro systems," says Pauline Gee, vice-president of predictive biology at MDS. Iconix's DrugMatrix database now includes more than 200 of these drug signatures, which are used by drug companies such as Bristol-Myers Squibb to help select candidate drug molecules more efficiently and to improve their understanding of the safety of new compounds before preclinical development. 